\title{
An X-ray spectrometry method for estimating diffusion and ion- exchange properties of rocks
}

\author{
SAMUEL MORFIN ${ }^{1}$, GOLROKH HAFEZIAN ${ }^{1}$, SAMUEL \\ $\mathrm{KUMAHOR}^{1}$, TOM AL ${ }^{*, 1}$ \\ ${ }^{1}$ Department of Earth and Environmental Sciences, \\ University of Ottawa, Ottawa, ON, K1N 6N5, Canada \\ [*correspondence: tom.al@uottawa.ca]
}

Measurements of diffusion and sorption properties of rocks are key aspects of site characterization for a deep geological repository. We present an X-ray spectrometric method for monitoring iodide and cesium $\left(\mathrm{I}^{-}\right.$and $\left.\mathrm{Cs}^{+}\right)$tracer migration in lab-scale diffusion experiments. This allows estimation of ion diffusion coefficients, cation exchange capacity (CEC) and ion-selectivity coefficients $\left(\log \mathrm{K}_{\mathrm{Cs}^{+}+\mathrm{Na}^{+}}\right)$for $\mathrm{Cs}^{+}$exchange.

The method is similar to X-ray radiography $[1,2]$ in its concept and advantages; it is non-destructive, rapid and it allows measurement of time- and spatially-resolved tracerconcentrations and spatially-resolved porosity. Analytical or numerical models may be used to fit the spatially-resolved concentrations to obtain the desired diffusion and ionexchange properties at multiple time increments during the transient phase of solute diffusion [2].

Measurements are conducted in X-ray transmission mode, whereby a collimated beam $(1 \mathrm{~mm}$ diameter in the current setup) is transmitted through a rotating cylindrical rock core. The beam is oriented normal to the rotation axis, effectively "sampling" a disk-shaped volume of the core with a height of $1 \mathrm{~mm}$. An energy-dispersive detector (Amptek XR-100-CdTe) records the transmitted X-ray spectrum. Starting with the $\mathrm{X}$ ray beam incident on either the top or bottom of the rock sample, a spectrum is acquired for a fixed time interval, typically 5 minutes. This is repeated in $1 \mathrm{~mm}$ steps along the length of the sample.

Rather than measuring total X-ray attenuation in the sample, as is done with radiography, spectrometry allows for measurement of attenuation only in a selected energy range. In the present work, measurements are conducted in specified energy ranges immediately below and above the $\mathrm{K}$ absorption edge for the tracer $\left(33.2 \mathrm{keV}\right.$ and $36.0 \mathrm{keV}$ for $\mathrm{I}^{-}$and $\mathrm{Cs}^{+}$ respectively). There are important advantages. The method virtually eliminates artifacts from beam hardening, it simplifies calibration and provides optimal sensitivity to the tracer.

[1] Cavé et al. (2009) J. Contam. Hydrol. 10(1-2), 1-12.

[2] Loomer et al. (2013) Applied Geochemistry 39, 49-52. 